THE French weekly geographical paper, La Géographie, has, after five years in the ordinary garb of a newspaper, assumed a new form, each number consisting of eight quarto pages in a coloured wrapper.

Mr. H. Yule Oldham, Lecturer on Geography in Owens College, Manchester, has been appointed to the lectureship on Geography in the University of Cambridge, formerly held by Mr. J. Y. Buchanan, F.R.S. Mr. Oldham has mainly studied the historical aspects of geography, and in his appointment the University of Cambridge obviously intends to associate its geographical teaching with the Historical rather than the Natural Science Board of Studies. It is to be hoped that the lectureship will receive more attention from the members of the University than has been given to it hitherto, and that the loss to scientific geography caused by Mr. Buchanan's retirement will be more than made up by increased interest in the less specialised aspects of the science.

MR. H. M. CADELL gives a remarkably interesting map of the site of Edinburgh in prehistoric times in the June number of the Scottish Geographical Magazine. The most noteworthy feature is the submergence of the 25 teet raised beach on which the greater part of Leith is now built, and the existence of seven comparatively large lakes of which the shrunken remnant only remain, or which have been ertirely drained and reclaimed within the historic period. A summary of the evidence for the existence of these lakes is given in the form of a short article. It is noteworthy that the changes in the surface of the land due to cultivation and building operations have in some cases almost entirely concealed the original features. In the early human period the shores of the Firth of Forth must have been occupied by a succession of swampy lakes dominated by the steep cliffs of the volcanic hills.

\section{SEISMOLOGY IN IAPAN.}

THE editor insists in a Wordsworthian manner on calling this the seventeenth volume although it is really vol. $i$. of the journal: he numbers it as a continuation of publications hitherto issued as the Transactions of the Seismological Society of Japan. The Society was founded in 1880 and for many years its meetings were frequent and well attended. It ceased to live in so far as subscriptions and meetings are concerned in I 892, many of its members having left the country. It may now be said to exist as much as ever it did, but without subscriptions. The transactions are in sixteen volumes of scientific papers to which a general index is published in this first number of the journal, and there can be no doubt of the great value of these papers, or of the ability and industry in experiment and speculation of the men who wrote them. During the twelve years' work of the Society much was accomplished; some order was evolved out of chaos; seismographs have been invented giving absolute measurements of earth motions, and a complete change has been effected in earthquake observation; a chair of seismology has been established in the Im. perial University and there is now a bureau controlling a central observatory and some 700 outside stations, together with many seismological laboratories. This is some of the work which the Society has done.

The first paper in this journal "On the Mitigation of Earthquake Effects, and Certain Experiments on Earth Physics" by the editor, reads very strangely to any one unacquainted with the work done by Prof. Milne in the last fifteen years. For example, on the construction of buildings in earthquake countries, his experiments have led to such results that he can speak with certainty on things which used to be merely matters of vague speculation, such as the security given by depth of foundation and the great differences in the earth motions at places within a few hundred yards of one another.

Probably no one can speak with greater authority on photographic matters than Prof. Burton, who contributes an article "On the Application of Photography to Seismology and Volcanic Pheromena." The other papers are :-An abstract of "The Seismometrical Observations for the year 1890," by the editor; "An Account of Experiments on the Overturning and Fracturing of Brick and other Columns by Horizontally Applied Motion," by the editor and Prof. Omori ; "On Earth Pulsations in relation to certain Natural Phenomena and Physical Investi-

1 The Seismological Journal of Japan, edited by John Milne, F.R.S. Vol. xvii. 1893 .

NO. I 232 , VOL. 48 ] gations," by the editor ; an abstract on observations by Dr. E. Von Rebeur-Paschwitz with horizontal pendulums; a note on old Chinese earthquakes, by Prof. Omori, and a note by the editor on the destructive earthquake of 1891 . All these papers seem to me to be valuable and interesting; they ought to be studied by every young philosopher whose mathematical and other weapons are ready, but who is yet without mental employment. The subject is one of world-wide interest, although it may seem to be only interesting to people like the Japanese who are jogged into attention every week of their lives.

The beautiful series of photographs published by Burton and Milne about a year ago are records that can never be branded as lies or exaggerations. Even Dr. Johnson, who to his dying day denied the fact that an earthquake had occurred at Lisbon, would have been convinced by records such as these. Without these photographs it would be difficult to believe in the actual compression in area of land over a large district or in vertical wave motion, travelling along a street as if the earth were water in a canal. The Japanese cannot neglect the study of the subject and other people ought not. Our time also may come, even in England, when in a five seconas interval, three fourths of all the houses in London may tumble into ruin and a quarter of a million sterling may be lost on every square mile of English ground. It is of no use to argue from the long histories of ancient cities. Earth shakes that had no evil effect on the more or less pyramidal architecture of Assyria and Egypt would lay the dwelling houses of London in long swathes upon the ground. One laughs at Alice's White Knight who was so well prepared for sharks, but we also laugh at Mrs. Aleslime whose specific in the real time of danger was "black stockings for sharks." Whatever our own safety may be we must remember that some of the most interesting parts of the world are vitally interested in this question, and the most artistic, most honest, most kindly, most generous and confiding clever people that the world has ever seen are demanding from us that we shall study this question to find out whatever means there may be for mitigating the effects of earthquakes, and more than all, taking away from them the dreadful everpresent feeling of danger, which seems in itself almost sufficient to arrest progress in civilisation.

We western people were till lately represented in Japan on this question by the Seismological Society. What one earnest worker and a few of his friends can do is being done, but in spite of earnestness and devotion, I am afraid that in one respect there must be a lessened result. The existence of the Society was of some weight in maintaining the interest of the Japanese Government on what must seem to non-scientific people a rather hopeless search for information. Even the small and exceed. ingly intermittent assistance of the British Association grant is of enormous moral value to Prof. Milne; and I think that if the council at Edinburgh had yielded to the representations of section $\mathrm{A}$ and granted the modest request of Prof. Milne for $£ 25$, they would have done more good than they can do with any equal sum in their present list. We have here a man who is untiring in experimental work, who has the power of keeping enthusiasm alive in other people to a remarkable degree, who is not a wealthy man and who yet spends some hundreds of pounds a year of his own, in making and using apparatus and in publishing a journal which has about seventeen subscribers. And all the work is good; it is thankless work as all work on the beginning of a science must be.

If every reader of NATURE who is interested in the matter and who can afford it, would only send to Prof. Milne a subscription (one pound a year) to this journal, his losses would be confined to his experimental work ; the Japanese Government would more certainly continue to interest its officials in making observations, and the subscribers would glow in the consciousness of having done their duty.

JOHN PERRY.

\section{ON LIGHT AND OTHER HIGH FREQUENCY} PHENOMENA. ${ }^{1}$

BRILLIANTLY worded, comprehensive, and strikingly illustrated was a lecture delivered by Mr. Nikola Tesla, of which a report has just reached us. In his own words :-

1 A lecture delivered before the Franklin Institute, at Philadelphia, Feb. 24, 1893, and before the National Electric Light Association, at St. Louis, Mo., March 1, 1893 . 
"In presenting these insignificant results I have not attempted to arrange and co-ordinate them as would be proper in a strictly scientific investigation in which every succeeding result should be a logical sequence of the preceding, so that it might be guessed in advance by the careful reader or attentive listener. I have preferred to concentrate my energies chiefly upon advancing novel facts or ideas which might serve as suggestions to others, and this may serve as an excuse for the lack of harmony. The explanations of the phenomena have been given in good faith, and in the spirit of a student prepared to find that they admit of a better interpretation. There can be no great harm in a student taking an erroneous view, but when great minds err, the world must dearly pay for their mistakes."

The following extracts will serve to show the character of the discourse :-

\section{The Action of the Eye.}

It can be taken as a fact, which the theory of the action of the eye implies, that for each external impression, that is, for each image produces upon the retina, the ends of the visual nerves concerned in the conveyance of the impression to the mind, must be under a peculiar stress or in a vibratory state. It now does not seen improbable that, when by the power of thought an image is evoked, a distinct reflex action, no matter how weak, is exerted upon certain ends of the visual nerves, and therefore upon the retina. Will it ever be within human power to analyse the condition of the retina when disturbed by thought or reflex action, by the help of some optical or other means of such sensitiveness, that a clear idea of its state might be gained at any time? If this were possible, then the problem of reading one's thoughts with precision, like the characters of an open book, might be much easier to solve than many problems belonging to the domain of positive physical science, in the solution of which many, if not the majority, of scientific men implicitly believs. Helmholtz has shown that the fundi of the eyes are themseives luminous, and he was able to see, in total darkness, the movement of his arm by the light of hi; own eyes. This is one of the most remarkable experiments recorded in the history of science, and probably only a few men could satisfactorily repeat it, for it is very likely that the luminosity of the eyes is associated with uncommon activity of the brain and great imaginative power. It is fluorescence of brain action, as it were.

Another fact havirig a bearing on this subject which has probably been noted by many, since it is stated in popular expressions, but which I cannot recollect to have found chronicled as a positive result of o sservation is, that at times, when a sudden idea or image presen:s itseif to the intellect, there is a distinct and sometimes painful sensation of luminosity produced in the eye, observable even in broad daylight.

Two facts about the eye must forcibly impress the mind of the physicist, notwithstanding he may think or say that it is an imperfect optical instrument, forgetting that the very conception of that which is perfect or seems so to him, has been gained through this same instrument. Firstly, the eye is, as far as our positive knowledge goes, the only organ which is directly affected by that subtile riedium, which, as science teaches us, must fill all space; secondly, it is the most sensitive of our organs, incomparably more sensitive to external impressions than any other.

This divine organ of sight, this indispensable instrument for thought and all intellectual enjoyment, which lays open to us the marvels of this universe, through whicn we have acquired what knowledge we possess, and which prompts us to, and controls, all our physical and mental activity. By what is it affected? By light! What is light?

It is beyond the scope of my lecture to dwell upon the subject of light in general, my object being merely to bring presently to your notice a certain class of light effects and a number of phenomena observed in pursuing the study of these effects. But to be consistent in my remarks it is necessary to state that according to that idea, now accepted by the majority of scientific men as a positive result of theoretical and experimental investigation, the various forms of manifestations of energy which were generally designated as "electric" or more precisely "electromagnetic" are energy manifestations of the same nature as those of radiant heat and light. Therefore the phenomena of light and heat, and others besides these, may be called electrical phenomena. Thus electrical science has become the mother science of all and its study has become all-important. The day when we shall know exactly what "electricity" is, will chronicle an event probably greater, more important than any other recorded in the history of the human race.

\section{Transformation of Currents.}

Mr. Tesla then went on to describe the apparatus employed, and the method of obtaining the high potentials and high frequency currents which are made use of in his experiments. In order to explain the transformation of currents he used the following analogy :-

Imagine a tank with a wide opening at the bottom, which is kept closed by spring pressure, but so that it snaps off suddenly when the liquid in the tank has reached a certain heigbt. Let the fluid be supplied to the tank by means of a pipe feeding at a certain rate. When the critical height of the liquid is reached, the spring gives way and the bottom of the tank drops out. Instantly the liquid falls through the wide opening, and the spring, reasserting itself, closes the bottom again. The tank is now filled, and after a certain time interval the same process is repeated. It is clear that if the pipe feeds the fluid quicker than the bottom outlet is capable of letting it pass through, the bottom will remain off and the tank will still overflow. If the rates of supply are exactly equal, then the bottom lid will remain partially open, and no vibration of the same and of the liquid column will generally occur, though it might, if started by some means. But if the inlet pipe does not feed the fluid fast enough for the outlet, then there will be always vibration. Again, in such case, each time the bottom flaps up or down, the spriny and the liquid column, if the pliability of the spring and the inertia of the moving parts are properly chosen, will perform independent vibrations. In this analogue the fluid may be likened to electricity or electrical energy, the tank to the condenser, the spring to the dielectric, and the pipe to the conductor through which electricity is supplied to the condenser. To make this analogy quite complete it is necessary to make the assumption, that the bottom, each time it gives way, is knocked violently against a non-elastic stop, this impact involving some loss of energy, and that, besides, some dissipation of energy results, due to frictional losses. In the preceding analogue the liquid is supposed to be under a steady pressure. If the pressure of the fluid be assumed to vary rhythmically, this may be taken as corresponding to the case of an alternating current. The process is then not quite as simple to consider, but the action is the same in principle.

\section{Electrostatic Force.}

After showing that the human body could be traversed by a powerful electric current vibrating at about the rate of one million times per second, Mr. Tesla said :-

The amount of energy which may thus be passed into the body of a person depends on the frequency and potential of the currents, and by making both of these very great, a vast amount of energy may be passed into the body without causing any discomfort except perhaps in the arm, which is traversed by a true conduction current. The reason why no pain in the body is felt, and no injurious effect noted, is that everywhere, if a current be imagined to flow through the body, the direction of its flow would be at right angles to the surface; hence the body of the experimenter offers an enormous section to the current, and the density is very small, with the exception of the arm perhaps, where the density may be considerable. But if only a small fraction of that energy would be applied in such a way that a current would traverse the body in the same manner as a low frequency current, a shock would be received which might be fatal. A direct or low-frequency alternating current is fatal I think, principally because its distribution through the body is not uniform, as it must divide itself in minute streamlets of great density, whereby some organs are vitally injured. That such a process occurs I have not the least doubt, though no evidence might apparently exist or be found upon examina. tion. The surest to injure and destroy life is a continuous current, but the most painful is an alternating current of very low frequency. The expression of these views, which are the result of long-continued experiment and observation, both with steady and varying currents, is elicited by the interest which is at present taken in this subject and by the manifestly erroneous ideas which are daily propounded in journals on this subject.

The electrostatic attractions and repulsions between bodies of measurable dimensions are, of all the manifestations of this force,

NO. I 232 , VOL. 48 ] 
the first so-called electrical phenomena noted. But though they have been known to us for many centuries, the precise nature of the mechanism concerned in these actions is still unknown to us, and has not been even quite satisfactorily explained. What kind of mechanism must that be? We cannot help wondering when we observe two magnets attracting and repelling each other with a force of hundreds of pounds with apparently nothing between them. We have in our commercial dynamos magnets capable of sustaining in mid-air tons of weight. But what are even these forces acting between magnets when compared with the tremendous attractions and repulsions produced by electrostatic force, to which there is apparently no limit as to intensity. In lightning discharges bodies are often charged to so high a potential that they are thrown away with inconceivable force and torn asunder or shattered into fragments. Still even such effects cannot com pare with the attractions and repulsions which exist between charged molecules or atoms, and which are sufficient to project them with speeds of many kilometres a second so that under their violent impact bodies are rendered highly incandescent and are volatilized. It is of special interest for the thinker who inquires into the nature of these forces to note, that whereas the actions between individual molecules or atoms occur seemingly under any condition, the attractions and repulsions of bodies of measurable dimensions imply a medium possessing insulating properties. So, if air, either by being rarefied or heated, is rendered more or less conducting, these actions between two electrified bodies practically cease, while the actions between the individual atoms continue to manifest themselves.

\section{Single-avire Transmission.}

It has been for a long time customary, owing to the limited experience with vibratory currents, to consider an electric current as something circulating in a closed conducting path. It was astonishing at first to realise that a current may flow through the conducting path even if the latter be interrupted, and $i$ was still more surprising to learn, that sometimes it may be even easier to make a current flow under such conditions than through a closed path. But that old idea is gradually dis appearing, even among practical men, and will soon be entirely forgotten.

It is thought useful to devote here a few remarks to the subject of operating devices of all kinds by means of only one leading wire. It is quite obvious, that when high-frequency currents are made use of, ground connections are at least, when the E.M.F. of the currents is great-better than a return wire. Such ground connections are objectionable with steady or low frequency currents on account of destructive chemical actions of the former and disturbing infuences exerted by both on the neighbouring circuits; but with high frequencies these actions practically do not exist. Still, even ground con nections become superfluous when the E.M.F. is very high, for soon a condition is reached when the current may be passed more economically through open, than through closed con ductors. Remote as might seem an industrial application of such single wire transmission of energy to one not experienced in such lines of experiment, it will not seem so to any one who for some time has carried on investigations of such nature. Indeed I cannot see why such a plan should not be practicable. Nor should it be thought that for carrying at such a plan currents of very high frequency are implicitly required, for just as soon as potentials of say 30,000 volts are used, the single wire transmission may be effected with low frequencies, and experiments have been made by me from which these inferences are made.

\section{Electrical Resonance.}

Some remarks and experiments were then made with regard to electrical resonance. Continuing, Mr. Tesla said :-

In connection with resonance effects and the problem of transmission of energy over a single conductor which was previously considered, I would say a few words on a subject which constantly fills my thoughts and which concerns the welfare of all. I mean the transmission of intelligible signals or perhaps even power to any distance without the use of wires. I am becoming daily more convinced of the practicability of the scheme, and though I know full well that the great majority of scientific men will not believe that such results can be practically and immediately realised, yet I think that all consider the developments in recent years by a number of workers to have been such as to encourage thought and experiment in this direction. My conviction has grown so strong that I no longer look upon this plan of energy or intelligence transmission as a mere theoretical possibility, but as a serious problem in electrical engineering, which must be carried out some day. The idea of transmitting intelligence witbout wires is the natural outcome of the most recent results of electrical investigations. Some enthusiasts have expressed their belief that telephony to any distance by induction through the air is possible. I cannot stretch my imagination so far, but I do firmly believe that it is practicable to disturb by means of powerful machines the electrostatic con dition of the earth, and thus transmit intelligible signals and perhaps power. In fact, what is there against the carrying out of such a scheme? We now know that electric vibration may he transmitted through a single conductor Why then not try to avail ourselves of the earth for this purpose? We need not be frightened by the idea of distance. To the weary uanderer counting the mile-posts the earth may appear very large, but to that happiest of all men, the astrononer, who gazes at the heavens and by their standard judges the magnitude of our globe, it appears very small. And so, I think, it must seem to the electrician, for when he considers the speed with which an electric disturbance is propagated through the earth all his ideas of distance must completely vanish.

A point of great importance would be first to know what is the capacity of the earth? and what charge does it contain if electrified? Though we have no positive evidence of a charged body existing in space without other oppositely electrified bodies being near, there is a fair probability that the earth is such a body, for by whatever process it was separated from other bodies - and this is the accepted view of its origin-it must have retained a charge, as occurs in all processes of mechanical separation. If it be a charged body insulated in space it s capacity should be extremely small, less than one-thousandth of a farad. But the upper strata of the air are conducting, anc: so, perhaps, is the medium in free space beyond the atmosphere, and these may contain an opposite charge. Then the capacity might be incomparably greater. In any case it is of the greatest importance to get an idea of what quantity of electricity the earth contains. It is difficult to say whether we shall ever acquire this necessary knowledge, but there is hope that we may, and that is by means of electrical resonance. If ever we can ascertain at what period the earth's charge, when disturbed, oscillates with respect to an oppositely electrified system or known circuit, we shall know a fact possibly of the greatest importance to the welfare of the human race. I propose to seek for the period by means of an electrical oscillator, or a source of alternating electric currents. One of the terminals of the source would be connected to earth, as, for instance, to the city water mains, the other to an insulated body of large surface. It is possible that the outer conducting air strata or free space contains an opposite charge and that, together with the earth, they form a condenser of very large capacity. In such case the period of vibration may be very low and an alternating dynamo machine might serve for the purpose of the experiment. I would then transform the current to a potential as high as it would be found possible and connect the ends of the high tension secondary to the ground and to the insulated body. By varying the frequency of the currents and carefully observing the potential of the insulated body and watching for the disturbance at various neigh. bouring points of the earth's surface resonance might be detected. Should, as the majority of scientific men in all prob. ability believe, the period be extremely small, then a dynamo machine would not do and a proper electrical oscillator would have to be produced and perhaps it might not be possible to obtain such rapid vibrations. But whether this be possible or not, and whether the earth contains a charge or not, and whatever may be its period of vibration, it certainly is possible-for of this we have daily evidence-to produce some electrical disturbance sufficiently powerful to be perceptible by suitable instruments at any point of the earth's surface.

\section{Production of Light.}

The light effects which it has been the chief object to investigate can be divided into four classes: (I) Incandescence of a solid. (2) Phosphorescence. (3) Incandescence or phosphorescence of a rarefied gas, and (4) Luminosity produced in a gas at ordinary pressure. The first question is, How are these luminous effects produced? In order to answer this question as satisfactorily as I am able to do in the light of accepted views and with the experience

NO. I 232 , VOL. 48 ] 
acquired, and to adil some interest to this demonstration, I shall dwell here upon a feature which I consider of great importance, inasmuch as it promises, besides, to throw a better light upon the nature of most of the phenomena produced by high frequency electric currents. I have on other occasions pointed out the great importance of the presence of the rarefierl gas, or atomic medium in general, around the conductor through which alternate currents of high frequency are passed, as regards the heating of the conductor by the currents. My experiments described some time ago have shown that the higher the frequency and potential difference of the currents, the more im. portant becomes the rarefied gas in which the conductor is immersed, as a factor of the heating. The potential difference, however, is, as I then pointed out, a mure important element than the frequency. When both of these are sufficiently high, the heating may be almost entirely due to the presence of the rarefied gas. [Experiments were performed showing the importance of the rarefied gas, or generally of gas at ordinary or other pressure as regards the incandescence or other luminous effects produced by currents of this kind.]

\section{Incandescent Lamps.}

Disregarding now the modifying effect of convection, there are two distinct causes which determine the incandescence of a wire or filament with varying currents, that is, conduction current and bombardment. With steady currents we have to deal only with the former of these two causes, and the heating effect is a minimum, since the resistance is least to steady flow. When the current is a varying one the resistance is greater, and hence the heating effect is increased. Thus if the rate of change of the current is very great, the resistance may increase to such an extent that the filament is brought to incandescence with inappreciable currents, and we are able to take a short and thick block of carbon or other material and bring it to bright incandescence with a current incomparably smaller than that required to bring to the same degree of incandescence an ordinary thin lamp filament with a steady or low frequency current. This result is important, and illustrates how rapidly our views on these subjects are changing, and how quickly our field of knowledge is extending. In the art of incandescent lighting, to view this result in one aspect only, it has been commonly considered as an essential requirement for practical success, that the lamp filament should should be thin and of high resistance. But now we know that the resistance to the steady flow of the filament does not mean anything; the filament might as well be short and thick; for if it be immersed in rarefied gas it will become incandescent by the passage of a small current. It all depends on the frequency and potential of the currents. We may conclude from this, that it would be of advantage, so far as the lamp is considered, to employ high frequencies for lighting, as they allow the use of shot and thick filaments and smaller currents.

If a wire or filament be immersed in a homogeneous medium, all the heating is due to true conduction current, but if it be enclosed in an exhausted vessel the conditions are entirely different. Here the gas begins to act and the heating effect of the conduction current, as is shown in many experiments, may be very small compared with that of the bombardment. This is especially the case if the circuit is not closed and the potentials of course very high. Suppose a fine filament enclosed in an exhausted vessel be connected with one of its ends to the terminal of a high tension coil and with its other end to a large insulated plate. Though the circuit is not closed, the filament, as I have before shown, is brought to incandescence. If the frequency and potential be comparatively low, the filament is heated by the current passing through it. If the frequency and potential, and principally the latter, be increased, the insulated plate need be but very small, or may be done away with entirely; still the filament will become incandescent, practically all the heating being then due to the bombardment. . . . It should not be thought that only rarefied gas is an important factor in the heating of a conductor by varying currents, but gas at ordinary pressure may become important, if the potential difference and frequency of the currents is excessive. On this subject I have already stated, that when a conductor is fused by a stroke of lightning, the current through it may be exceedingly small, not even sufficient to heat the conductor perceptibly, were the latter immersed in a homogeneous medium.

NO. I 232 , VOL. 48 ]
From the preceding it is clear that when a conductor of high resistance is connected to the terminals of a source of high frequency currents of high potential, there may occur considerable dissipation of energy, principally on the ends of the conductor, in consequence of the action of the gas surrounding the conductor. Owing to this, the current through a section of the conductor at a point midway between its ends may be much smaller than throatgh a section near the ends. Furthermore, the current passes principally through the outer portions of the conductor, but this effect is to be distinguished from the skin effect as ordinarily inter. preted, for the latter would or should occur also in a continuous incompressible medium. If a great many incandescent lamps are connected in series to a source of such currents, the lamps at the ends may burn brightly, whereas those in the middle may remain entirely dark. This is due principally to bombardment, as before stated. But even if the currents be steady, provided the difference of potential is very great, the lamps at the ends may burn more brightly than those in the middle. In such case there is no rhythmical bombardment, and the result is produced entirely by leakage. This leakage or dissipation into space, when the tension is high, is considerable when incandescent lamps are used, and still more considerable with arcs, for the latter act like flames. Generally, of course, the dissipation is much smaller with steady than with varying currents.

\section{Incandescence of Gases.}

Coming now to the incandescence or phosphorescence of gases at low pressures or at the ordinary pressure of the atmosphere, we must seek the explanation of these phenomena in shocks or impacts of the atoms. Just as molecules or atoms beating upon a solid body excite phosphorescence in the same or render it in. candescent, so when colliding among themselves they produce similar phenomena. But this is a very insufficient explanation, and concerns only the crude mechanism. Light is produced hy vibrations which go on at a rate almost inconceivable. If we compute, from the energy contained in the form of known radiations in a definite space the force which is necessary to set up such rapid vibrations, we find, that though the density of the ether be incomparably smaller than that of any body we know, even hydrogen, the force is something surpassing comprehension. What is this force, which in mechanical measure, may amount to thousands of tons per square inch? It is electrostatic force in the light of modern views. It is impossible to conceive how a bod of measurable dimensions could be charged to so high a potential that the force would be sufficient to produce these vibrations. Long before any such charge could be imparted to the body it would be shattered into atoms. The sun emits light and heat, and so does an ordinary flame or incandescent filament, but in neither of these can the force be accounted for if it be assumed that it is associated with the body as a whole. Only in one way may we account for it, namely, by identifying it with the atom. An atom is so smalt, that if it be charged by coming in contact with an electrified body and the charge be assumed to follow the same law as in the case of bodies of measurable dimensions, it must retain a quantity of electricity which is fully capable of accounting for these forces and tremendous rates of vibration. But the atom behaves singularly in this respect, it always takes the same "charge."

It is very likely that resonant vibration plays a most important part in all manifestations of energy in nature. Throughout space all matter is vibrating, and all rates of vibration are represented, from the lowest musical note to the highest pitch of the chemical rays, hence an atom, or complex of atoms, no matter what its period, must find vibration with which it is in resonance. When we consider the enormous rapidity of the light vibrations, we realise the impossibility of producing such vibrations directly with any apparatus of measurable dimensions and we are driven to the only possible means of attaining the object of setting up waves of light by electrical means and economically, that is, to affect the molecules or atoms of a gas, to cause them to collide and vibrate.

Much would remain to be said about the luminous effects produced in gases at low or ordinary pressures. With the present experiences before us we cannot say that the essential nature of these charming phenomena is sufficiently known. But investigations in this direction are being pushed with exceptional ardour. Erery line of scientific pursuit has its fascinations, but electrical investigation appears to possess a peculiar attraction, for there is no experi- 
ment or observation of any kind in the domain of this wonderful science which would not forcibly appeal to us. Some beautiful experiments with a vacuum tube concluded the lecture.

\section{UNIVERSITY AND EDUCATIONAL INTELLIGENCE.}

CAMBRIDGE.-The Observatory Syndicate have prepared a report with respect to the future work of the Cambridge Observatory.

They are of opinion that in the present condition of Astronomy provision must be made for photographic work, so that their inquiries have been directed to the discovery of the best scheme for rendering the Northumberland Equatorial available in connection with the project for a photographic establishment. The primary question as to the respective merits of reflectors and refractors for the photographic instrument had of course to be considered. On this issue there is considerable difference of opinion. For producing representations of astronomical objects, where great detail is required, reflectors are the most suitable. But where accurate measurement of the photographic plates is the object in view the balance of opinion seems clearly to show that the refractor is better adapted than the reflector. As it seems obvious that the work undertaken at the Cambridge $\mathrm{Ob}$ servatory should be based on accurate measurement the Syndicate have come to the conclusion that the refractor is the photographic telescope that should be employed. They think it right, however, to draw the attention of the University to the kindness of Mr. Common who offered to make and present to the Observatory a suitable silvered glass mirror if it were decided to employ the reflecting instrument.

It may be well to add that the Newall Telescope is devoted in the main to spectroscopic work and further that this instrument having been made for visual observation is not adapted to the special photographic work to which it is now proposed to direct the energies of the Observatory.

The scheme which the Syndicate suggest is that a new objective of eighteen inches aperture corrected for the photographic rays be provided ; that the focal length of this should be about the same as that of the Northumberland objective, for which a new tube will be required; and that the two objectives, united as a pair like the present instruments at Greenwich and Oxford, should be erected on a new mounting, under a new dome, in the building at present occupied by the Northumberland Equatorial.

It will be observed that, by this scheme, the Northumberland objective will still be useful for every purpose for which it has been hitherto employed, with the great additional advantages of an excellent mounting and a good clock work. For example, such observations of comets as have been previously made here can be conducted under circumstances of much greater convenience than before. As to the special work to be undertaken by photography, it appears to the Syndicate that for the present under the particular conditions in which work here can be conducted there is no subject so promising as stellar parallax. The Director of the Observatory desires to undertake a systematic search with the aid of photography for stars which have measurable parallax, and of course so complete an apparatus as is now proposed would be available for many other researches besides that just suggested.

A preliminary estimate for the new telescope and mounting complete makes the cost $£ 2450$. To this must be added $£ 500$ for the new dome, and $\delta 150$ for the measuring apparatus. If $£$ IOo be added for extras this makes a total of $€ 3200$. There is now a sum of about $£ \mathrm{r} 500$ in the Special Sheepshanks Fund available for the purchase of instruments. In view of future contingencies, to exhaust the Sheepshanks Fund would be un advisable and indeed it would not suffice for the purchase of an I8-inch equatorial. As such an instrument would contribute largely to the astronomical services of the Observatory the Syndicate think that an appeal to the public for subscriptions would probably be successful and such an appeal they are prepared to make.

It is therefore recommended that they be authorised to obtain estimates and plans for a new instrument as above described.

Dr. Hill, Master of Downing College, has been appointed a representative of the University at the International Medical Congress to be held at Rome next September.

\section{SCIENTIFIC SERIALS.}

American Meteorological Fournal, May.-The following are the nrincipal meteorological articles:-Meteorology as the physics of the atmosphere, by Prof. W. v. Bezold. This is a translation by Prof. C. Abbe of the first part of an important paper from Himmel and Erde. It describes the problems which at present are the subject of theoretical investigation, and points out what new problems have grown from looking at observational meteorology from a theoretical point of view. During the last decade attention has been chiefly devoted to the development of the so-called convection theory, which is principally based on observations at the earth's surface, but which, at higher elevations, is found to have defects. It has therefore become necessary to try and connect this theory with that of the old trade-wind theory, which for several decades has been entirely set aside. More attention is required to observations made in the higher regions of the atmosphere, together with the application to them of the principles of general mechanics, as well as of thermo-dynamics.-Charts of storm frequency, by Prof. Abbe. The author has plotted in a tabular form the number of storm centres that pass over each quadrangular degree between lat. $20^{\circ}$ and $49^{\circ} \mathrm{N}$., and long. $99^{\circ}$ and $63^{\circ} \mathrm{W}$., deduced from the tridaily Signal Service charts, from March, $187 \mathrm{r}$, to February, 1873. He states that the chart from which the table is prepared clearly shows that the storm tracks, which move from Alberta and Assinniboin south-eastward over the United States and then north-eastward towards the gulf of St. Lawrence, describe a system of parabolic curves whose tendency is to have a common point of intersection, and therefore a region of maximum storm frequency, in, or to the north-west of Nebraska. - Six and seven day weather periodicities, by $\mathrm{H}$. $\mathrm{H}$. Clayton. The author, who has studied the subjects of periodicities for several years, found a striking regularity between the intervals of many of the temperature maxima of the Blue Hill observations, and that almost all the maxima could be arranged in such a way that they followed each other at intervals of six or seven days. He thinks that, for a large part of the year, forecasts of temperature, on the assumption of regular rhythmic oscillations, and a knowledge of the time of their beginning and ending, may be made for a week or two in advance with nearly as much accuracy as they are now made by the Weather Bureau for thirty-six hours.

American fournal of Mathematics, vol. xv., No. 2. (Baltimore, April, I893). - The opening memoir is one entitled "Hyperelliptische Schnittsysteme und Zusammenordnung der Algebraischen und Transcendentalen Thetacharacteristiken," by H. D. Thompson (pp. 91-123). There are numerous figures and an index of contents. - On the determination of groups whose order is a power of a prime, by J. W. A. Young (pp. I 24-I 78), considers in some detail groups of the order specified in extension of the work on groups by Cayley $(\mathrm{Am}$. F. of Math., vol. i.), Kempe (Phil. Trans., vol. clxxvii.), Netto (Substitutionentheorie, pp. I33-7), and Kronecker. The author's aim has been " to presuppose no knowledge of the theory of groups on the part of the reader."-The third paper, the projection of fourfold figures upon a three-flat, by T. P. Hall (pp. 179-189), is an interesting contribution to the literature of higher space, and the last page (190) contains a note on a geometrical theorem by C. N. Little. It gives a property of a Pascal line, and a Brianchon point of 6 gons formed in a specified manner.

Wiedemann's Annalen der Physik und Chemie, No. 5.-On electrical discharges: production of electrical oscillations and their relation to discharge tubes, by H. Ebert and E. Wiedemann. This portion of the work investigates the manner in which the properties of the conducting circuit determine the sensibility of the discharge-tube when placed in a given position with regard to the terminal condenser. Among the conditions thus studied were the distance between the plates of the primary condenser, the D.P. in the primary spark.gap, and the frequency of the sparks; also the influence of bridges across the wire system, the D.P. required to make the tubes glow, and the effects of the presence of other glowing tubes in the field. As regards the last, it was found that if a glowing gas was present in a portion of the condenser field the distribution of energy was quite different from that in a homogeneous field; the tubes of energy were attracted towards the gas and passed through it, showing that the gas in the state of glow has a greater per-

NO. I 232 , VOL. 48] 